\title{
Infrared Micro-Thermography of High-Power AllnGaN LEDs Using High Emissivity (black) in IR and Transparent in the Visible Spectral Region Coating
}

\author{
by A. Aladov*, A. Chernyakov*, A. Zakgeim* \\ *Submicron Heterostructures for Microelectronics Research and Engineering Center of RAS, 26 \\ Politekhnicheskaya, 194021, St.Petersburg, Russian Federation, zakgeim@mail.ioffe.ru
}

\begin{abstract}
We report a technique which can simplify and improve the Infrared (IR) micro-thermography of light-emitting diodes (LEDs) by the use of a special thin-film coating which combines strong absorption in the wavelength range of sensitivity of IR microscope $(2.5-3 \mu \mathrm{m})$ and transparency in the visible region of the own LED radiation. As is well known, conventional IR temperature measurements, made on the LEDs, lead to significant difficulties and errors as consequence of the optical transparency of substrate/heterostructure layers and low emissivity of metalized contacts. The proposed method allows avoiding the time-consuming procedure of emissivity calibration for different materials forming LEDs, while increasing the measurement accuracy.
\end{abstract}

\section{Introduction}

High-power and efficient LEDs become the main electronic components of modern lighting technology. This process is accompanied by a continuous growth of LED output optical power, achieved by increasing operating currents, the chip area with complicating geometry of the electrodes and mounting pads. Accordingly, there is a significant increase in the heat released and self-heating of devices. At the same time, the emission efficiency and degradation rate of LEDs depend heavily on the temperature of the active area. It should be noted that today, for high-quality LEDs, heat is the critical factor limiting their capabilities. Recently, a large number of papers have been devoted to the problem of LED thermal management [1]. Conventionally, the thermal properties of LEDs, like other semiconductor devices, are characterized by thermal resistance. The latter is determined from measurements of temperature-sensitive parameter (forward voltage, emission wavelength, etc.) under excitation by a current of a special form [2]. The main drawback of parameter thermal resistance is that it allows to estimate only the average temperature over the whole area of a LED active region. This 39is correct, however, only in the specific case when the generated heat distribution inside the chip is uniform. More commonly, configuration of the LED electrodes, aimed at minimizing the shading effect, leads to the current crowding, especially at high excitation levels. The current crowding entails the non-uniform heat generation, respectively, the non-uniform temperature distribution on the chip. Since local temperature and current density have a significant influence on the quantum efficiency the real temperature distribution (temperature map) seems to be especially important for operating of the advanced high-power LEDs.

Among the methods for determining the thermal properties of LEDs, the most informative is IR-microthermography, which allows to estimate not only device average heating, but also to establish the temperature distribution over the area with the detection of overheat spots and temperature gradients (temperature mapping) [3]. For large-area high-power LEDs this is especially important in connection with the effects of current crowding, light absorption and heat release under contacts. Unfortunately, with respect to LEDs, the IR thermal imaging microscopy is significantly complicated due to difference between the black body and materials forming the LED: transparent layers of sapphire (or silicon carbide) substrate, AllnGaN heterostructures and highly reflective (low emissivity) metalized contacts. Significant efforts should be made to pre-calibrate the IR microscope when working with different materials, but still cannot avoid the errors associated with calibration [2]. The simple solution - deposition of high emissivity coating on the test surface is unacceptable for LED due to the absorption of own LED emission and distortion of the real heat generation and dissipation pattern (It should be noted that in modern LEDs, light takes up to $70 \%$ of the input power). Additionally, the conventional coating excludes the possibility of optical measurements, in particular, the near-field of electroluminescence (EL), necessary for the full characterization of the device. As a more successful approach, one can consider the thermography method using carbon micro-particles occupying part of the surface and having properties close to the black body [3]. However, in this case, there is also a partial distortion of the heat dissipation pattern due to the absorption of the LED emission by the carbon particles. In addition, a temperature map becomes discrete.

To overcome these shortcomings, we propose new technique of IR temperature measurements by employment the special thin-film coating close to the optical properties of black body in the range of sensitivity of IR microscope and transparent in the visible region of the own LED emission. Thus, the real mode of LED operation and measuring its electro-optical parameters are preserved, but acquired the opportunity for effective thermal mapping. 


\section{Experiment}

\subsection{Samples and their electro-optical characteristics}

Commercial Vertical LED chips SL-V-B45AK (peak emission wavelength $\lambda_{p}=470 \mathrm{~nm}$ ), produced by SemiLEDs Corporation [4] were used in the experimental study. LED chips had the geometric dimensions of the square $1050 \times 1050$ $\mu \mathrm{m}^{2}$, a total thickness of $145 \mu \mathrm{m}$ and represented a multi-layered epitaxial AllnGaN heterostructure thickness of about 5 $\mu \mathrm{m}$ transferred by techniques "lift-off" and "bonding" on a copper substrate. Advantages of the copper substrate in comparison with the initial sapphire due to the high electrical and thermal conductivity. The schematic view of the chip is shown in the Fig. 1. As can be seen the chip has a contour-strip top electrode to provide current spreading while minimizing shading of the output light. The chip was soldered on silicon submount, which in turn was mounted on a bulky aluminium radiator.
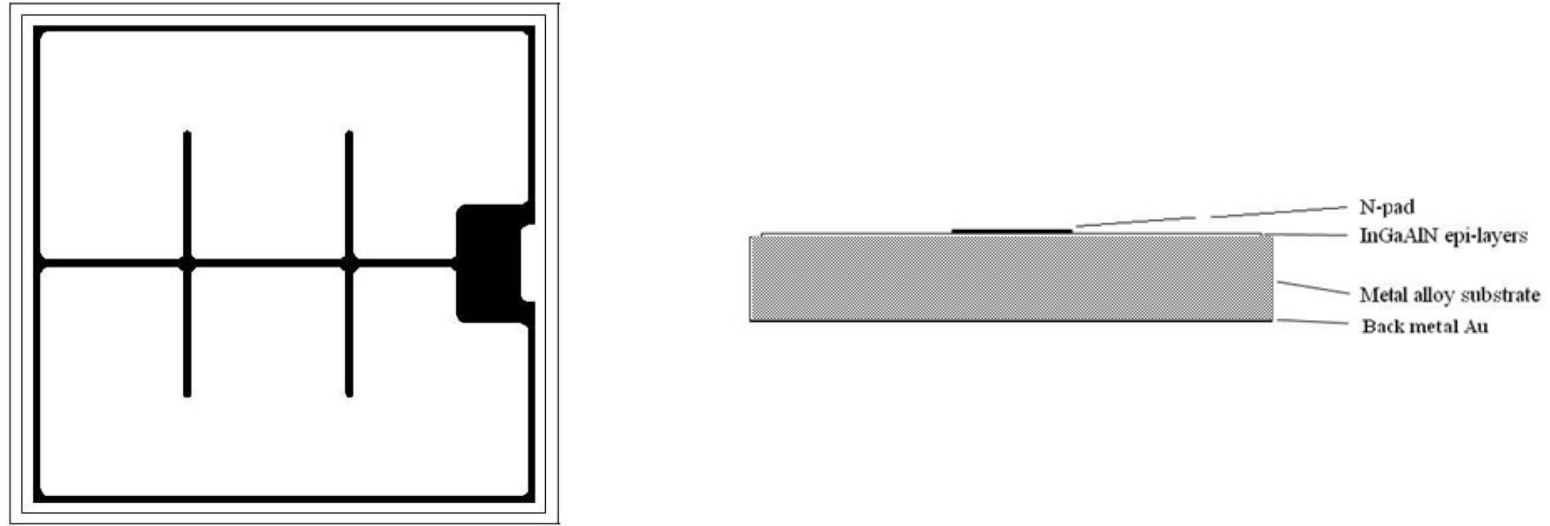

Fig. 1. Schematic chip design: Electrode configuration is shown by black color.

Previously, before conducting the IR thermal imaging, basic electro-optical LED characteristics were measured. Light-current characteristics with evaluation of the output optical power (optical cooling) was obtained using the "OL 770 LED High-speed LED Test and Measurement System" (Optronic Laboratories, Inc.). The thermal resistance was measured by the forward-voltage relaxation method with the "Thermal Transient Tester T3Ster" (MicRed, Ltd.). The nearfield of the electroluminescence (EL) was mapped by a Mitutoyo optical microscope equipped with a Canon digital camera (12 Mpxl CMOS photo-sensitive matrix) with the best spatial resolution of $0.25 \mu \mathrm{m}$. For example, the experimental EL emission pattern obtained at the current of $I=1 \mathrm{~A}$ is shown in Fig. 2a as a colour contour chart (in view of the symmetry only the lower half of the chip is represented). The profile of the EL intensity in the cross section A, indicated by dashed line, is shown in Fig. $2 \mathrm{~b}$. The above measurements are necessary to establish the proportion of heat carried away by the own emission as also for the subsequent comparison of the temperature and EL distributions over the LED area. As will be shown below, a comparative analysis of light and temperature distributions enables to receive more information about the internal processes in LEDs (quantum efficiency and its dependence on current density and temperature).

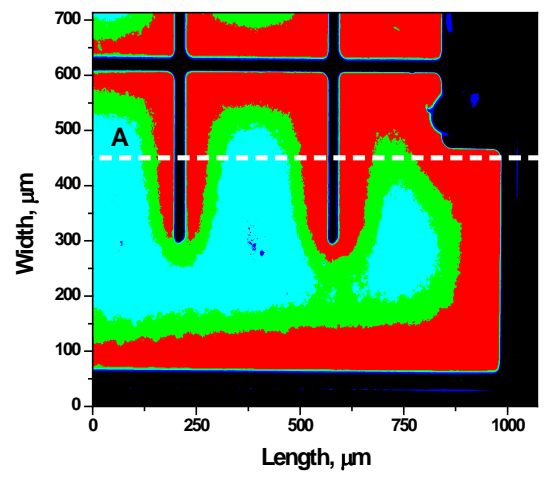

a

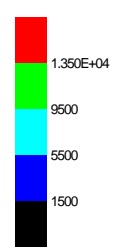

Fig. 2. Contour plot showing near-field $E L$ intensity distribution at $I=1 \mathrm{~A}$ (a) and profiles of $E L$ intensity in the cross-
section $A$, indicated by dash line (b)

Fig. 2. Contour plot showing near-field EL intensity distribution at $I=1 \mathrm{~A}$ (a) and
section $A$, indicated by dash line (b)

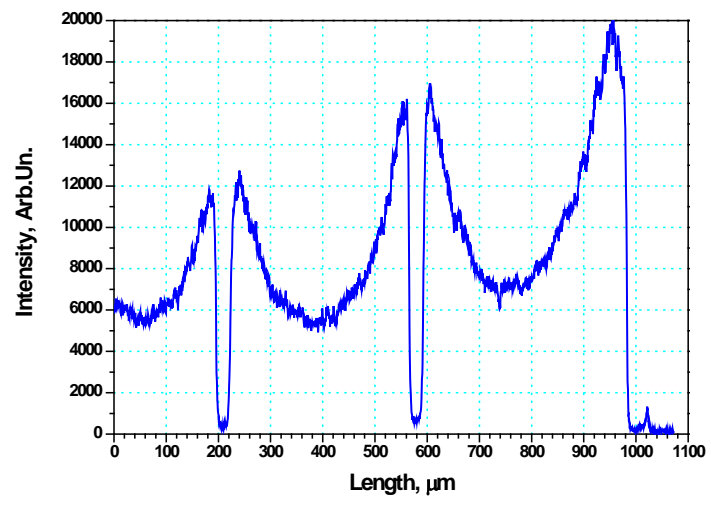

b 


\subsection{IR thermal imaging: equipment and method}

There is a wide diversity of IR thermal imaging systems intended for evaluating the temperature of objects on the basis of their intrinsic thermal radiation in the spectral range from 3 to $12 \mu \mathrm{m}$. However, these systems are mostly applicable to macroscopic objects. In the present study, we employed a UTK-1 infrared (IR) microscope specially designed for analysis of temperature distributions in semiconductor chips [5]. Photo of the microscope with the control interface is shown in Fig. 3. The thermal radiation was recorded with an InAs photodetector matrix: $128 \times 128$ cells (sensors) with a $50 \mu \mathrm{m}$ step between them. As shown in Fig. 4 (curve 2), for the InAs photodetector the sensitivity region lies in the wavelength range 2.5-3.1 $\mu \mathrm{m}$. It should be noted that using a relatively short wavelength sensitivity range compared with the region 5-12 $\mu \mathrm{m}$ utilized in conventional thermal imaging systems leads to an improvement the spatial resolution up to diffraction limit $\sim 3 \mu \mathrm{m}$. The temperature resolution about $0.2 \mathrm{~K}$ is achieved for a black body at $300 \mathrm{~K}$.

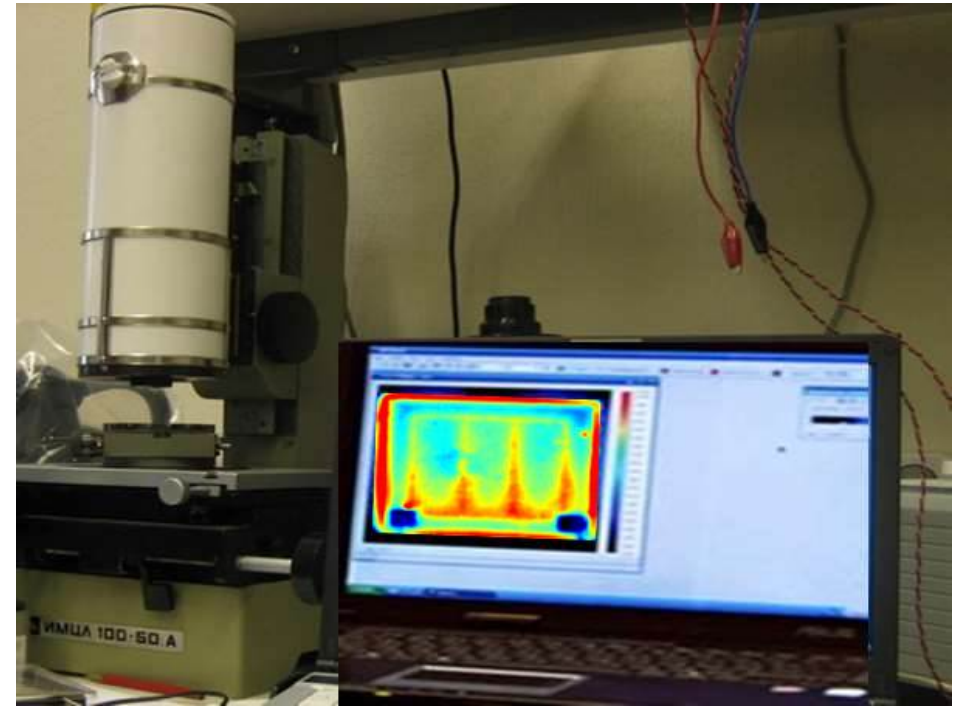

Fig. 3. General view of the UTK-1 IR microscope system with the interface.

The main methodological problems of thermal imaging of LED structures are (i) the transparency of the sapphire substrate and epitaxial layers for IR radiation and (ii) a large difference in the emissivity of the materials utilized in the LED, i.e., semiconductor layers, metallic electrodes, reflective coatings, mounting elements, etc. [6]. Therefore to extract the correct temperature values from the IR images requires preliminary calibration for each particular material. Initially, the temperature of all LED structural units is maintained by an external heater with simultaneous digital recording of the IR emission intensity (bits). The "emission intensity-temperature" calibration curve is then used to determine the absolute temperatures of the objects in real operation mode of the LED. The calibration procedure is time-consuming, moreover, leads to additional errors in obtaining of temperature maps, especially at points of low emissivity.

As already mentioned, the application of absorbent coatings, even partial in the form of micro-particles, is unacceptable for LEDs due to the absorption of own visible emission and disturbance of real thermal and optical regimes of operation. In order to avoid this obstacle, for the IR temperature mapping of LEDs we propose a film coating having a high transparency in the visible region and a high absorption in the sensitivity region of the IR microscope. Such "IRblack - VIS-transparent" film is based on inorganic pigment and epoxy resin and is applied to the surface of the device by pouring and subsequent centrifugation up to a thickness of about $40 \mu \mathrm{m}$ providing sufficient absorption in the IR region. Fig. 4 shows the spectral dependence of the film transmission (thickness of $40 \mu \mathrm{m}$ ) in the visible and IR spectra against of the emission spectra of LEDs and the sensitivity range of our IR microscope. As can be seen from Fig. 4 (curve 1), the film has an almost perfect transparency (T>95\%) in the whole visible region of the spectrum of $0.4-0.8 \mu \mathrm{m}$ used by LEDs and significant absorption in the sensitivity range of 2.5-3.1 $\mu \mathrm{m}$ of the IR microscope. The maximum absorption coefficient is attained at a wavelength of $2.9 \mu \mathrm{m}$ and is $k \sim 550 \mathrm{~cm}^{-1}$, the integral absorption coefficient over the entire spectral sensitivity region of the microscope is $k_{\text {int }} \sim 350 \mathrm{~cm}^{-1}$. As illustrated in Fig. 5 (curve 2), such strong absorption provides high values of the emissivity of the "IR-black - VIS-transparent" film, which is approaching the InAs with antireflective coating used by us as a black body simulator. 


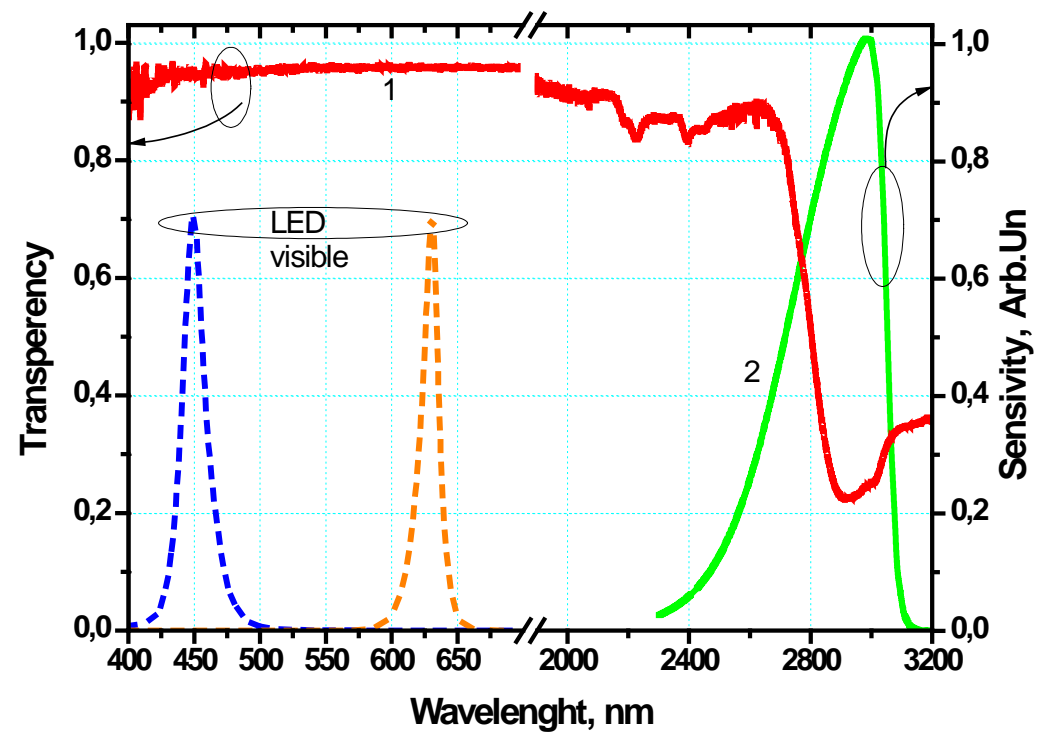

Fig. 4. Transmission spectrum of "IR-black - VIS-transparent" film (1) on the background of spectral sensitivity of the IR microscope (InAs; 77K) (2). The dashed line shows the typical emission spectra of the LED

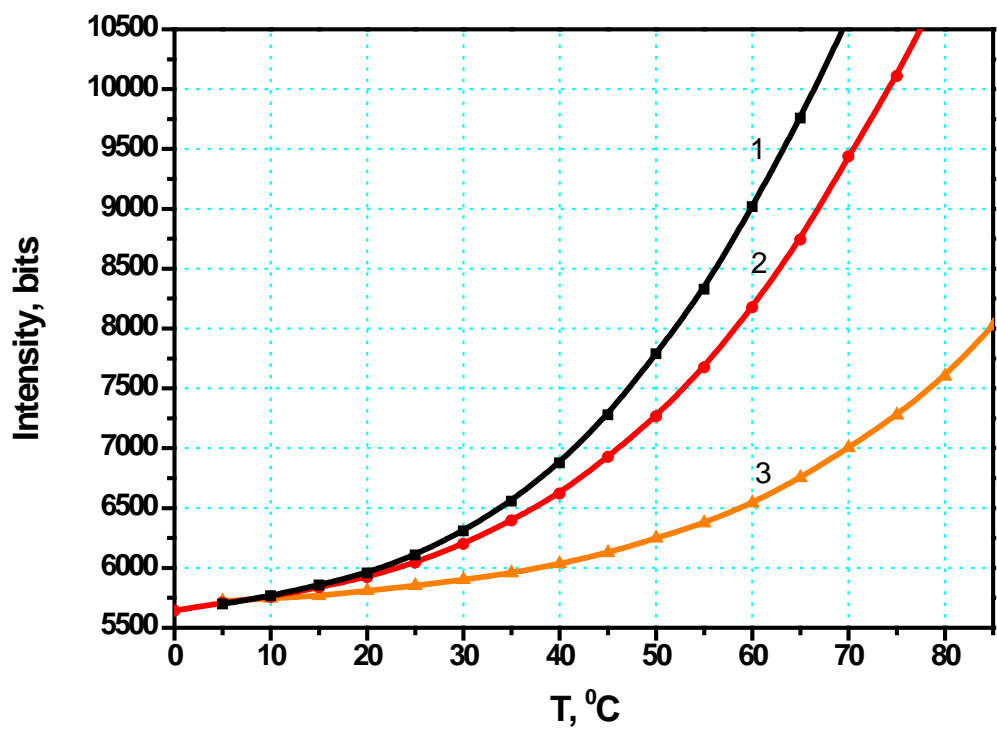

Fig. 5. Plots of IR emission intensity versus temperature (nonlinear calibration curves) for various materials: InAs with antireflective coating as a black-body simulator (1), "IR-black - VIS-transparent" film (2), Au contact (3)

\subsection{Thermal measurement}

Fig. 6a,b shows IR microphotographs (in other words, IR emission intensity distribution) for an LED chip SL-VB45AK at $l=1$ A before and after coating with a "IR-black - VIS-transparent" film. As can be seen, the strong color contrast in Fig. 6a is determined not so much by the temperature distribution as by the difference in the emissivity and reflectivity of the LED elements: the strip top contact, the bottom contact, the semiconductor layers and the welding points. In order to convert Fig. 6 a to a temperature map, the intensity at each point must be multiplied by the calibration factor corresponding to the material at this point. Completely different image appears in Fig. $6 \mathrm{~b}$ : metallic and semiconductor layers are no longer visible through the IR-opaque film. The absolute value of the signal has increased significantly and the color contrast reflects the purely temperature distribution of the black surface with a uniform emissivity (the dark spot on the left is contact welding not covered with a film). 


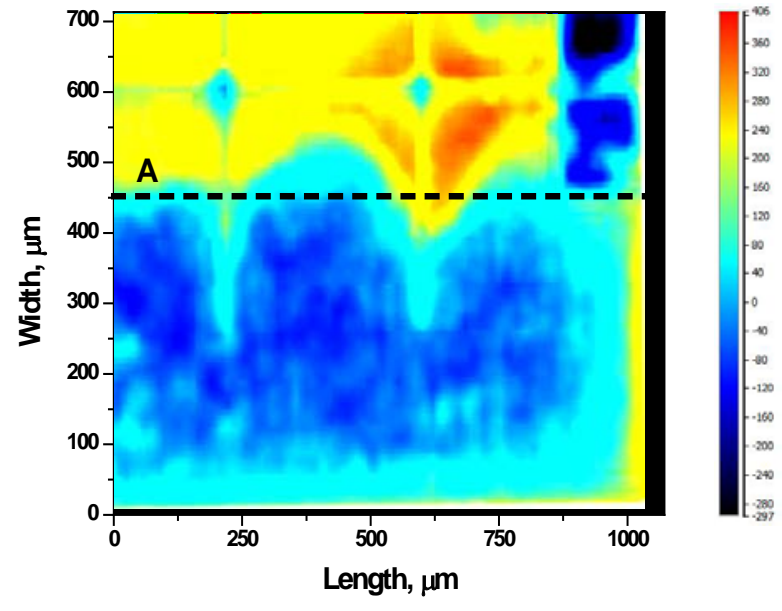

a

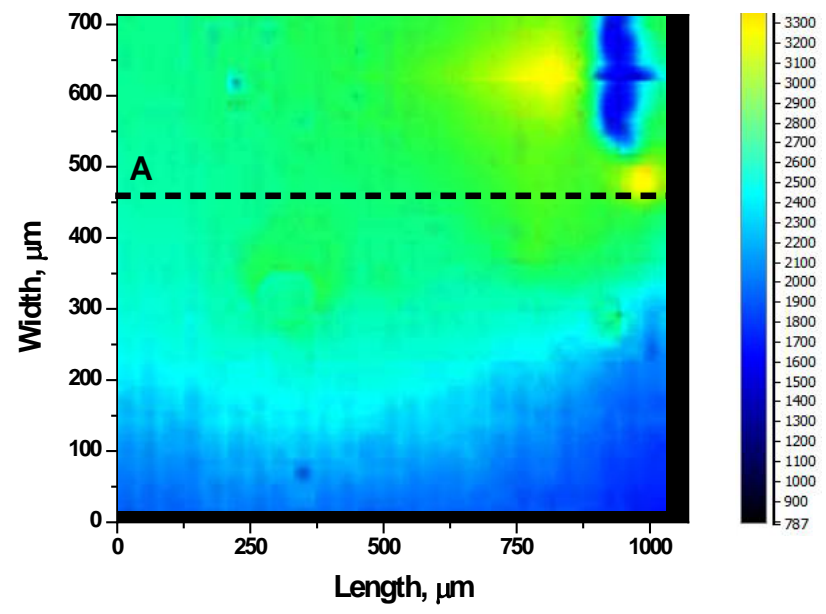

b

Fig. 6. IR image of LED at bias current I = 1 A for bare chip (a) and chip with "IR-black - visible-transparent" coating (b)

\section{Results and discussion}

Based on Fig. 6b, we obtain the corresponding temperature distribution in cross section A, i.e. the local temperature profile in the LED chip, shown in Fig. 7, curve 1. This profile is easily calculated from the distribution in Fig. $6 \mathrm{~b}$ taking into account the calibration (curve 2 in Fig. 5). It is interesting to compare the distribution of temperature and current (temperature and current density profiles) in the emitting chip. It should be noted that the observed EL intensity profile cannot be considered an accurate reflection of the current density distribution profile. This is due both to the dependence of the internal quantum efficiency on the current density, and photon propagation, multiple reflections and scattering inside the LED chip. Therefore, it can be argued that non-uniformity of the EL intensity distribution between the contacts and from edge to edge along the section A (Fig.7, curve 2) remains smoother than the current density distribution but reflects the latter in the first approximation [7]. On the other hand, the heat generation rate in the active region is roughly proportional to the current density and it would seem possible to expect a certain similarity in the distribution of temperature and current density. However, the experiment shows otherwise. A comparison of the temperature and EL profiles reveals that the temperature and current density distribution patterns are very different. As curve 1 in Fig. 7 shows, a very small temperature change $\sim 3^{0} \mathrm{C}$ is observed from the edge with a higher current density (near the contact welding) to the opposite edge with a lower density, without temperature gradients between vertical stripes of the electrodes. The latter is attributed to intensive lateral heat transfer in the Cu submount, thickness about 150 $\mu \mathrm{m}$ used in this LED design instead of a remote sapphire substrate. Achieving such a high uniformity in the temperature distribution indicates a successful thermal management of the LED chip providing not only the uniformity of temperature distribution but low values of total thermal resistance. It should be noted that such high thermal characteristics distinguish exactly the design of the LEDs under consideration. For other design versions ("flip-chip" or "face-up" on a sapphire substrate) at high currents, the effects of current crowding and significant local overheating are typical.

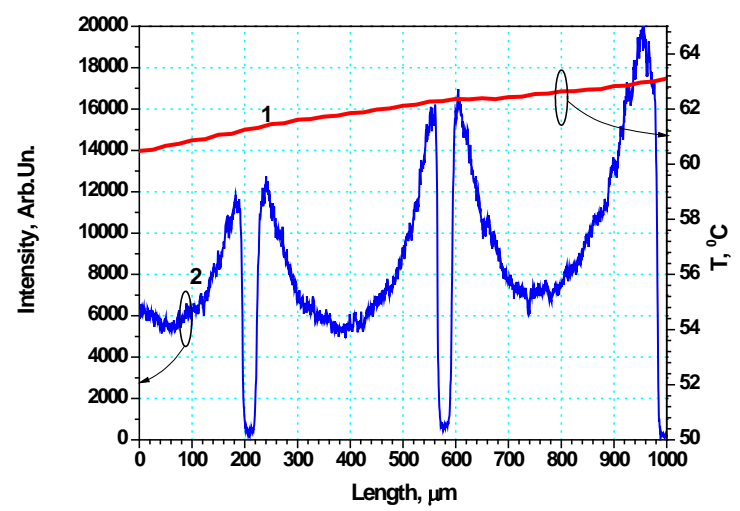

Fig. 7. Experimental temperature (1) and EL intensity (2) profiles over the cross section A measured at current $I=1$ A. 


\section{Conclusions}

We have demonstrated a novel technique to improve IR temperature mapping of visible high-power AIGalnN, AIGalnP LEDs. The method is based on the use of high emissivity thin film coating placed in isothermal contact with a device surface. A feature of the coating is transparency in the visible region of the spectrum and strong absorption in the IR region of sensitivity of the IR microscope ("IR-black - VIS-transparent" film). The combination of these properties allows to avoid absorption of the own LED emission and thus not disturb the real optical and thermal LED operation. At the same time, thanks to the high emission in the IR region, it is possible to eliminate difficulties and errors normally associated with low emissivity of metal electrodes and transparency of semiconductor materials. The coating technique is relatively simple and includes pouring and centrifugation.

By the proposed method, we obtained accurate maps of temperature distributions for high-power LED chips of large area with a branched geometry of the electrodes. Significant interest for the analysis electrical, thermal and optical processes into LEDs is a comparison of the temperature and EL intensity distribution. Taking into account that the local internal quantum efficiency at a given point depends on both current density and temperature, one can reveal the dominant negative factors affecting the operation of LEDs. From comparison the high-resolution maps of temperature and EL intensity for vertical LED chips SL-V-B45AK we have established noticeable effects of current crowding, but high uniformity of the temperature distribution. This means that due the replacement of a low thermal conductivity sapphire by a high thermal conductivity copper submount intensive heat transfer in the lateral directions is achieved.

\section{REFERENCES}

[1] Cl.J. M. Lasance, Andras Poppe. "Thermal Management for LED Applications" Springer, NY, 2014, 551 p.

[2] V. Szekely, A new evaluation method of thermal transient measurement results, Microelectronics journal, 28 , 277(1997)

[3] A. Kozlowska, M.Latoszek, et al. "Analysis of thermal images from diode lasers: Temperature profiling and reliability screening“, Appl. Phys. Lett. 86, 203503 (2005)

[4] SemiLEDs, Product Datasheet. Available: www.semileds.com

[5] V.M. Bazovkin, A.A. Guzev, A.P. Kovchavtsev, et al. "Thermal imaging microscope", Prikl.Fiz. 2, 97 (2005)

[6] A. L. Zakgeim, G. L. Kuryshev, M. N. Mizerov, V. G. Polovinkin, I. V. Rozhansky and A. E. Chernyakov. "Study of Thermal Processes in High Power InGaN/GaN Flip-Chip LEDs by IR Thermal Imaging Microscopy" Semiconductors 44, (3),373, (2010)

[7] A. E. Chernyakov, K. A. Bulashevich, S. Yu. Karpov, and A. L. Zakgeim. "Experimental and theoretical study of electrical, thermal, and optical characteristics of InGaN/GaN high-power flip-chip LEDs", Phys. Status Solidi A, 1-4 (2013) 